\title{
Color considerations in fluorescent solar concentrator stacks
}

\author{
Paul D. Swift and Geoff B. Smith
}

\begin{abstract}
We present modeled results of the luminous and color outputs of a three-layer stack of fluorescent planar concentrators (FPCs). FPCs have the potential to provide sufficient luminous output to illuminate moderate-sized rooms for reasonably-sized collecting areas. It is of course necessary not only that the lumens be sufficient, but also that the light be sufficiently white as to be comfortable. Modeling shows that by use of a stack of three FPCs, one each of violet, green, and red, it is possible to achieve good color rendering and sufficient lighting levels for room illumination. (c) 2003 Optical Society of America

OCIS codes: $350.6050,160.2540$.
\end{abstract}

\section{Introduction}

Fluorescent planar concentrators (FPCs) consist of a matrix that is highly transparent such as specialized glass or, more commonly for cost reasons, polymethyl methacrylate impregnated with a fluorescent dye. FPCs typically have a much greater width (approximately a few centimeters) and length (approximately many centimeters) than thickness (approximately a few millimeters). Radiation incident onto the largearea surface of the FPC is absorbed and reemitted by the fluorescent dye, and because the same quantity of radiation reaches each of the faces of the $\mathrm{FPC}$, it is concentrated at the small-area faces. The history of FPCs is closely linked with solar cells ${ }^{1}$; in a typical arrangement solar cells would be placed along the thin edge of an FPC that would then be illuminated with radiation that is considerably concentrated over that which would be obtained in direct sunlight. Use of an FPC provides nominal concentrations of the incident intensity at the small-area faces of $\sim 10^{2}$. However numerous considerations reduce this value, the most important of which are losses due to only a fraction of emitted radiation being totally internally reflected by the FPC, dye self-absorption, matrix

The authors are with the Department of Applied Physics, University of Technology, Sydney, P. O. Box 123, Broadway, New South Wales 2007, Australia. P. D. Swift's e-mail address is Paul.Swift@uts.edu.au.

Received 6 January 2003; revised manuscript received 22 May 2003.

$0003-6935 / 03 / 255112-06 \$ 15.00 / 0$

(C) 2003 Optical Society of America losses, dye quantum efficiency, nonabsorption of solar radiation by the dye, and geometric losses.

The possibility of an FPC as a source of light for room illumination is an idea that has been around for some time ${ }^{2,3}$ but has yet to be realized in practice. Numerous practical considerations need to be overcome-not least the stability of the dyes in continuous sunlight and the luminous efficiency of any proposed system. Perhaps a less pressing consideration, but no less important, is the color of the light that any FPC system provides, which needs to be near neutral. In the only illumination system known by the authors to have been reported in the literature, ${ }^{3}$ orange fluorescent dye was used to illuminate a small room.

The usefulness of an FPC is governed by its efficiency, the definition of which depends on the application. In the case of an FPC being used with photovoltaic cells, the efficiency is usually defined in terms of the ratio of the energy of the incident radiation to the electrical energy generated with radiation collected at the edges. In the case of the FPC being used as a source of light, the definition of efficiency is more usefully defined as a ratio of the lumens incident onto the collector to that collected at one of the edges (so-called luminous efficiency). The critical factors affecting the luminous efficiency of an FPC are the self-absorption and quantum efficiency of the fluorescent dye. High quantum efficiency organic dyes are readily available. The selfabsorption of the dye is due to the overlap of the emission spectrum and the absorption spectrum. Self-absorption not only reduces the output lumens but also influences the color of the emitted light, which consequently depends on the collector dimen- 
sions. The dye concentration is an important parameter in these considerations. Although it is possible to produce white light with two colors, such a light source will have poor rendering qualities; therefore, for all practical purposes a three-color system needs to be used.

An additional parameter that describes the quality of a source of light is the luminous efficacy, which is defined as the lumens per watt of a source. For the sun, through air mass 1.5 , the luminous efficacy is approximately $110 \mathrm{~lm} / \mathrm{W}$. The greater the efficacy of a source the greater the relative amount of light of a source lies in the visible part of the spectrum. Further, low-efficacy sources, used, for example, in office buildings, place additional loads on cooling, and so, clearly, high-efficacy sources are desirable.

A typical illuminance on a clear day can be taken as $10^{5} \mathrm{~lx}$. Because the eye can operate over a wide range of illuminance (a typical room illumination is a few hundred lux) only a small fraction of the lumens that this figure represents needs to be brought into a room to give sufficient light to be of practical use. For example, an FPC $0.1 \mathrm{~m}^{2}$ in area would only need to operate at $10 \%$ luminous efficiency to provide a $1000 \mathrm{~lm}$, which is approximately the output of a $100-\mathrm{W}$ incandescent light.

In this paper the color of the output of a three-layer FPC system is calculated and discussed with respect to the color luminous output, efficacy, and color rendering. The dyes used are some of the Lumogen dyes manufactured by BASF-namely, Lumogen 570 (violet), Lumogen 083 (green) and Lumogen 300 (red). The whiteness of the output light is quantified with the Commission Internationale de l'Eclairage $2^{\circ}$ chromaticity coordinates. The luminous efficiency depends on the spectral output that, if constrained to be white, will not be the maximum possible value obtainable for green light. The ability of an illuminating source to render the true color of an illuminated object is measured by the color-rendering index (CRI) of the source. One calculates the CRI by averaging the reflected (or transmitted) spectrum from eight standard colors and comparing these averages with those of a blackbody at the same color temperature as the source. The higher the value of the CRI (which has a maximum value of 100 ), the higher the ability of the source to show the true color of an object.

\section{Theory}

The arrangement of interest is that in which the dye is embedded in a matrix that is a rectangular prism, as shown in Fig. 1(a). The quantity that needs to be calculated is the radiant, and thence luminous, flux arriving at the collection edge. Let the fluorescent dye have a normalized (with respect to area) emission power spectrum $\varepsilon_{0}(\lambda)$, where $\lambda$ is the wavelength and in which the subscript is used to emphasize that it is the spectrum of the dye and not that of an FPC. Consider a dye molecule that is a distance $l$ from the collection edge as shown in Fig. 1(a). The dye molecule emits isotropically so that some light is emitted into solid angles that do not contribute to the radiant

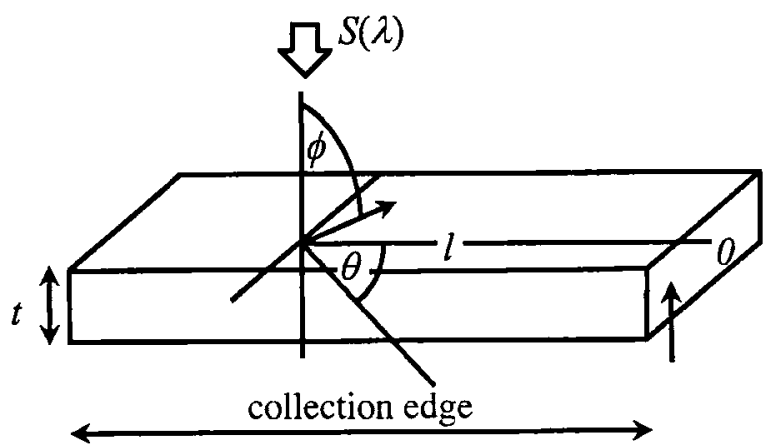

$L$

(a)

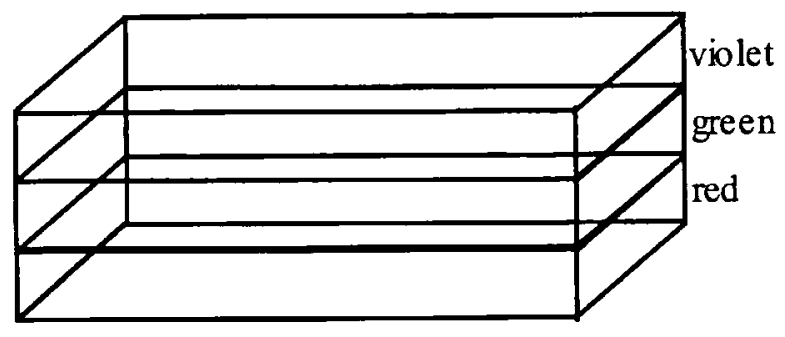

(b)

Fig. 1. (a) Coordinate system for a fluorescent solar concentration illuminated by a spectrum $S(\lambda)$. (b) Arrangement of three-layer stack modeled in this study.

flux at the collection edge. If light is totally internally reflected off both the top and the side surfaces, then it will reach the collection edge and will be available to be used as illumination. The form of the spectrum at the collection edge is then

$$
\begin{aligned}
\varepsilon(\lambda, l) \sim & \varepsilon_{0}(\lambda) \int_{x}^{\pi / 2} \mathrm{~d} \phi \sin \phi \int_{0}^{\operatorname{asin}(\cos x / \sin \phi)} \mathrm{d} \theta \\
& \times \exp \left[-\left[\alpha(\lambda)+\alpha_{m}\right] l /(\sin \phi \cos \theta)\right],
\end{aligned}
$$

where the angles $\phi$ and $\theta$ are standard spherical coordinates, $\alpha(\lambda)$ is the absorption spectrum at some nominal concentration of the dye in a particular matrix, $\chi$ is the critical angle of the matrix material, and $\alpha_{m}$ represents any matrix losses. As indicated in Expression (1), the condition for total internal reflection off the top and the bottom faces of the FPC is defined by the angle $\phi$, whereas both the spherical coordinates determine total internal reflection off the side face. If the refractive index of the matrix material is greater than 2, then Expression (1) needs to be modified for $\chi<\phi<\pi / 2-\chi$, in which range the upper limit of $\theta$ is $\pi / 2$. (If the refractive index of the matrix is less than 2 , then radiation incident at the top surface at the critical angle will not necessarily be totally internally reflected at the side surface. For materials of practical interest this is not a concern.) For an FPC of length $L$ the spectral intensity at the collection edge of the collector that results from the 
illumination of the sheet by an external source with spectral power $S(\lambda)$ is

$$
\begin{aligned}
\varepsilon(\lambda, L)= & \varepsilon_{0}(\lambda) \frac{E_{A}}{t(\pi / 2)} \int_{0}^{L} \mathrm{~d} l \int \mathrm{d} \phi \sin \phi \\
& \times \int \mathrm{d} \theta \exp \left\{-\left[\alpha(\lambda)+\alpha_{m}\right] l /(\sin \phi \cos \theta)\right\},
\end{aligned}
$$

where $t$ is the thickness of the collector, and for the case of normal incidence, the absorbed energy that is available for reemission by the dye (i.e., not lost by thermal processes), $E_{A}$, is

$$
E_{A}=(1-R) \int\{1-\exp [-\alpha(\lambda) t]\} S(\lambda) \eta_{e}(\lambda) \mathrm{d} \lambda,
$$

where $R$ is the reflectance of one surface of the collector and $\eta_{\mathrm{e}}$ the energy-conversion efficiency of the dye. The reflection from the base surface of the FPC back into itself represents only a small fraction of the energy available for absorption and can be safely neglected. Implicit in Eq. (2) is the well-known observation that the emission spectrum of a fluorescent material is independent of the excitation wavelength. ${ }^{4}$ Equation (2) is essentially that of previous researchers, ${ }^{1,5}$ except that the limits over the angle $\theta$ in those studies is in the range $[0, \pi / 2]$. This is justified in the geometric arrangement used with photovoltaic cells since the cells usually line the long edge of the FPC. In the geometry used in lighting, where the collectors are much longer than they are wide, light is expected to be collected from a shorter length edge, in which case this approximation is not valid.

The quantum (photon-photon) efficiency, $\eta_{q}$, which is typically a known parameter of a fluorescent material, is related to the energy conversion (energyenergy) efficiency $\eta_{e}$ via

$$
\eta_{e}(\psi)=\frac{\psi}{\int \lambda \varepsilon_{0}(\lambda) \mathrm{d} \lambda / \int \varepsilon_{0}(\lambda) \mathrm{d} \lambda} \eta_{q},
$$

where $\psi$ is the excitation wavelength. In the modeling presented here the output of the FPC is presented in terms of energy, and so Eq. (4) is needed. It is assumed in this study that the quantum efficiency is independent of excitation wavelength. 4

Equation (2) does not include any output component from self-absorbed radiation, and this fact can be taken into account by the following argument. If there was no self-absorption, then the spectral intensity would be given by placement of $\alpha(\lambda)=0$ in the argument of the angular integral of Eq. (2). Selfabsorbed radiation generates an additional source of radiation, and since the emission spectrum is independent of the excitation wavelength, then this absorbed radiation can be considered an additional energy source absorbed by the dye molecules. For the case of radiation that is emitted into the volume that would reach the collection edge of the FPC, this additional absorbed energy per unit area of the collector is

$$
2 \eta_{e} \frac{t}{L} \int(\varepsilon(\lambda, L ; \alpha=0)-\varepsilon(\lambda, L)) \mathrm{d} \lambda .
$$

The factor of 2 arises because of the oppositely directed volume into which radiation is emitted. For the volumes through which radiation would reach the top and the side surfaces, there are the additional energy densities of

$$
2 \eta_{e} \int[\varepsilon(\lambda, t ; \alpha=0)-\varepsilon(\lambda, t)] \mathrm{d} \lambda,
$$

and

$$
2 \eta_{e} \frac{w}{L} \int[\varepsilon(\lambda, w ; \alpha=0)-\varepsilon(\lambda, w)] \mathrm{d} \lambda,
$$

respectively. The inclusion of these terms in the energy incident on to the FPC is an upper estimate since it neglects further conversion losses. The value of $\eta_{e}$ can be taken as a constant whose value can be calculated at the wavelength where the product of the absorption and emission is maximum.

The quantity $\alpha_{\mathrm{m}}$ is typically small for the matrix, which must be highly transparent; to be otherwise would preclude their practical use, since light would be unable to travel any significant distance through the collector before being lost because of absorption. The magnitude of $\alpha_{m}$ may increase when a dye is introduced in such a way to give rise to scattering due to, for example, incomplete dissolution of the dye, which would result in colloidal scattering. In this paper it will be assumed that matrix losses are negligible. This approximation will overestimate the luminous output of the collector but will have little effect on the color of the emitted light of each of the FPC's.

To predict the spectral and luminous outputs of an FPC it is necessary to know the emission and absorption spectra of the dye in the collector. The dyes used in this research were the BASF dyes, Lumogen 300 (red), Lumogen 083 (green), and Lumogen 570 (violet), whose quantum efficiencies are 0.96, 0.91, and 0.92 , respectively. ${ }^{6}$ These well-known dyes are commercially available and have good photostability. Results of measurement and modeling of single FPCs have been published previously ${ }^{7}$ in which it was found that good agreement was found between modeled spectra and those measured for 1.2-m long collectors. This good agreement allows the confident computation of the output of a stack of collectors. To determine the spectral output of any stack of FPCs it is only necessary to determine the modified spectrum (i.e., modified from the solar spectrum through air mass 1.5 ) that illuminates the collectors that sit below any others. In a practical system it is expected, although not necessary, that the order of the FPCs 
would be violet, green, and red, which is the arrangement, shown in Fig. 1(b), modeled here. Each of the collectors would have a mirror on the edge opposite the collection edge, and the entire stack would sit on a base mirror. In this paper this is the arrangement considered, and two passes, through the stack to the mirror and back through the stack, of the illuminating source are taken into account. The mirrors are taken as perfect, which is a reasonable approximation since mirrors with reflectance values of 0.96 are readily available.

The luminous output of an FPC critically depends on the absorption spectrum of the dye even in the case of small absorption. Because the length of the FPC is of the order of a meter, then low values of the attenuation coefficient can have a significant effect on the luminous output of the FPC. Variations of one part in a thousand in the attenuation coefficient, which are at the limit of the accuracy of typical spectrophotometers, are significant. These considerations are detailed in a recent paper. ${ }^{8}$ The approach taken here is to take the point at which the attenuation spectrum is measured to be a minimum as zero, which will possibly result in the output being an overestimate for the 1-m-long collectors modeled in this study.

\section{Results}

Figure 2 shows the predicted luminous output of $1.0 \mathrm{~m} \times 0.1 \mathrm{~m} \times 0.002 \mathrm{~m}$ FPCs for individual collectors for each of the dyes in poly methyl methacrylate as a function of dye concentration. Curves are shown for the cases of a mirror at the end opposite the collection edge and of a mirror at the base of the collector and for cases with both mirrors present and with no mirrors present. The general shape of the curves can be understood in term of Eqs. (2) and (3). The integral term of Eq. (2) is a decreasing function of concentration, because the self-absorption of the dye increases with concentration, that is, a slowly decreasing function of concentration for moderate concentration values. The integral in Eq. (3) is an increasing function of concentration, because the absorbed energy increases with dye concentration, which saturates for moderate concentration values. The product of these functions will then have a maximum at some dye concentration, $c_{m}$ say, which depends on the degrees of absorption and selfabsorption of the dye. The result of adding a base mirror is to effectively increase the concentration with respect to the absorption of incoming radiation but not to the degree of self-absorption so that Eq. (3) rises more steeply with increasing concentrations for low concentration, whereas the integral term of Eq. (2) does not change. This result is a decrease in the value of $c_{m}$. The result of adding an edge mirror is to increase the integral in Eq. (2) without altering the absorbed energy so that again $c_{m}$ decreases. Adding both mirrors increases the degree of these effects, and the concentration of dye at which the maximum output occurs moves to a lower value than is the case for either mirror alone.
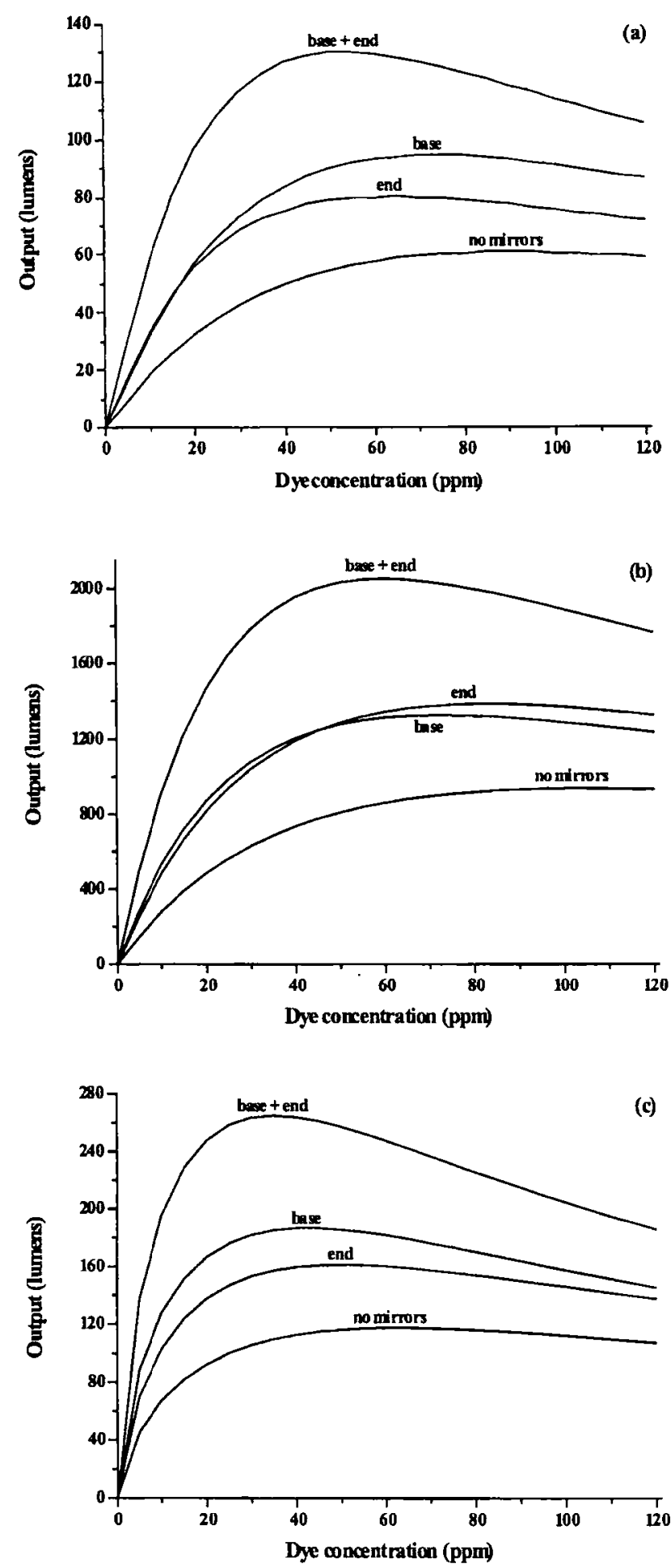

Fig. 2. Luminous outputs of single fluorescent solar collectors with and without mirrors present for (a) violet, (b) green, (c) red dye.

In the design of an FPC stack for use in daylighting the above results have a bearing on what concentrations need to be used. Any practical system uses end mirrors, on all single FPCs, and a base mirror. The base mirror used in a stack will have little effect on the top (violet) collector; thus its maximal output is at 


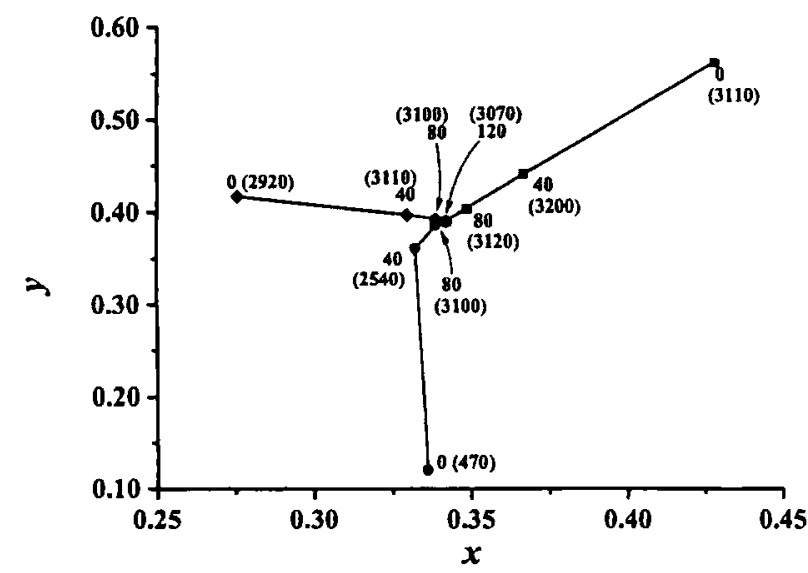

Fig. 3. Behavior of the color of a three-layer fluorescent solar concentrator with varying concentration of violet $(\boldsymbol{\square})$, green $(\bullet)$ and red $(\bullet)$ dyes. The dye concentration and luminous output (in parentheses) are indicated. The nonvarying concentration is 120 ppm in all cases.

a concentration near the case for the use of a rear mirror, which is also partially the case for the middle (green) collector. The concentrations that maximize the luminous output of each of the individual collectors are influenced by the concentration of the dyes used in each of the other collectors. The output is further complicated because the absorption spectra of the dyes overlap so that absorption in a collector leads to a reduced output in other collectors; and although one can maximize the luminous output of a collector within the stack by increasing the concentration of the dye, there may be a reduction in the total luminous output of the stack. As a starting point the combination of violet, green, and red, each at a concentration of $120 \mathrm{ppm}$ (by weight), provides a luminous output of approximately $3100 \mathrm{~lm}$ and with the chromaticity coordinates, $(x, y)$, equal to $(0.342$, $0.390)$. These coordinates represent a yellowishgreen source, which is reflected in the CRI of 58 and the high efficacy of $226 \mathrm{~lm} / \mathrm{W}$. It is possible to produce light whose color coordinates are within the chromaticity color triangle whose apexes are the color coordinates of the three collectors that make up the stack. For the violet, green, and red collectors of the dimensions used here and at the concentrations that give the maximum luminous output, the chromaticity coordinates are $(0.153,0.046),(0.349,0.638)$, and $(0.723,0.277)$ respectively. Stacks with coordinates near the apex represented by the green collector have larger luminous outputs, whereas those near the coordinates $(0.333,0.333)$ have more neutral, and lower, outputs. The chromaticity coordinates and luminous outputs of three-layer stacks with varying concentrations are shown in Fig. 3, which shows the importance of the green collector to the luminous output and that neutral light is possible with a threelayer stack but at the cost of reduced luminous output. Because it is the green collector that provides the greatest number of lumens per unit energy, it is this collector that needs to have its output reduced

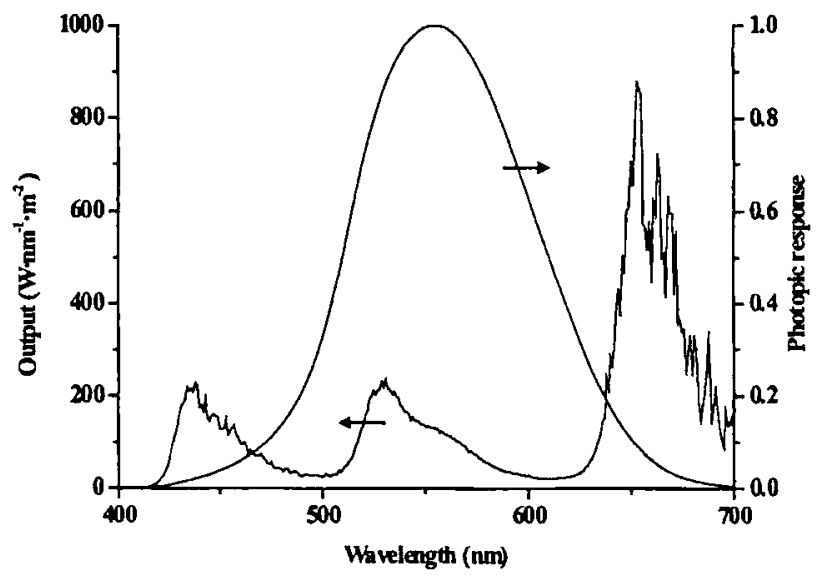

Fig. 4. Spectrum of the three-layer fluorescent solar concentrator whose color coordinates are $(0.333,0.333)$ with violet, green, and red collectors with concentration 95,24 , and $160 \mathrm{ppm}$, respectively, overlayed with the photopic response of the human eye.

while those of the red and violet collectors are maximized. One approach to maximizing the output of neutral light is first to maximize that of red light and to balance this light with violet and green. Such a process results in a luminous output of $2000 \mathrm{~lm}$ for a spectrum with chromaticity coordinate $(0.333,0.333)$ with violet, green, and red collectors with concentration 95,24 and $160 \mathrm{ppm}$, respectively. The CRI of this stack is 44 , a significantly lower figure than that for the stack that has $(x, y)=(0.342,0.390)$ described above. The spectrum of this collector is shown in Fig. 4, overlayed with the photopic response of the eye, which indicates the reason for the poor color rendering-namely, that the spectrum contains significantly lesser parts of blue and red wavelengths when compared with that of the green. The color rendering is poor because that rendering is increased in value as a spectrum becomes flatter across the visible. The efficacy of the output of this stack is approximately $160 \mathrm{~lm} / \mathrm{W}$, a higher figure than that of the solar spectrum air mass 1.5 .

\section{Conclusion}

It has been shown by modeling that a three-layer stack of FPCs can provide neutral-colored light of sufficient luminous intensity to illuminate a small room. The modeled dimensions of $1.0 \mathrm{~m} \times 0.1 \mathrm{~m} \times$ $0.002 \mathrm{~m}$ are of practice size. The luminous output is proportional to the width, but not to the thickness and the length, and increases in this dimension would be practicable. The modeling has not included any structure in which the FPC stack would need to be housed that would reduce the output by approximately $8 \%$ because of Fresnel reflection and whose spectral transmittance may influence the color, and hence luminance, of the light output.

Although the whiteness of the stack has been referenced to $(0.333,0.333)$ a wide range, chromaticity coordinate values are acceptable - for example, the sunlight with air mass 1 has color coordinates $(0.331$, 
0.344), whereas a standard white warm fluorescent lamp has coordinates $(0.437,0.405)$, which results in a greater luminous output and improved color rendering. ${ }^{9}$ The color rendering and luminous output for an equally neutral spectrum could be improved significantly by the use of a blue dye and a shorterwavelength orange-red dye. Unfortunately, blue dyes with high quantum efficiency are not available. However, a shorter-wavelength orange-red dye has recently become commercially available, and the output of a stack containing this dye will be an improvement over that modeled here. This dye has yet to be measured and modeled as a single FPC, which needs to be done prior to modeling it as part of a three-layer stack. There is also the possibility, at the expense of complicating the stack, of using a yellow dyed collector in tandem with the red collector so that each is a fraction of the total length of the stack. The yellow dye, which is commercially available, would increase both luminous output and color rendering of the dye.

\section{References}

1. W. H. Weber and J. Lambe, "Luminescent greenhouse collector for solar radiation," Appl. Opt. 15, 2299-2300 (1976).

2. S. Selkowitz, M. Rubin and B. Stearns, "Assessment report: fluorescent concentrators for daylighting applications," Internal Report LBL-15377 (Lawrence Berkeley Laboratory, Berkeley, 1982).

3. A. Zastrow and V. Wittwer, "Daylighting with fluorescent concentrators and highly reflective silver-coated plastic films: a new application for new materials," in Optical Materials Technology for Energy Efficiency and Solar Energy Conversion V, C. Grangvist, C. M. Lambert, and J. J. Mason, eds., Proc. SPIE 653, 93-100 (1986)

4. M. D. Lumb, Luminescence Spectroscopy (Academic, London, 1978).

5. J. S. Batchelder, A. H. Zewail, and T. Cole, "Luminescent solar concentrators. 1: Theory of operation and techniques for performance evaluation," Appl. Opt. 18, 3090-3110 (1979).

6. G. Seybold and G. Wagenblast, "New perylene and violanthrone dyestuffs for fluorescent collectors," Dyes Pigm. 11, 303-317 (1989).

7. P. D. Swift, G. B. Smith, and J. Franklin, "Light to light efficiencies in luminescent solar concentrators," in Solar Optical Materials XVI, C. M. Lampert and C.-G. Grangvist, eds., Proc. SPIE 3789, 29-34 (1999).

8. A. A. Earp, G. B. Smith, P. D. Swift, and J. Franklin, "Maximising the light output of a luminescent solar concentrator," submitted to Sol. Energy.

9. G. Wyszeski and S. Stiles, Color Science, 2nd ed. (Wiley, New York, 1982). 